\title{
Development of Simple Sequence Repeat Markers in Chinese Chestnut and Their Characterization in Diverse Chestnut Cultivars
}

\author{
Eiichi Inoue ${ }^{1}$, Lin Ning, and Hiromichi Hara \\ College of Agriculture, Ibaraki University, 3-21-1 Chuou, Ami-machi, Ibaraki 300-0393, Japan \\ Shuan Ruan \\ Division of Breeding, Liaoning Province Economic Forest Research Institute, Dalian, Liaoning, \\ China \\ Hiroyuki Anzai \\ Gene Research Center, Ibaraki University, 3-21-1 Chuou, Ami-machi, Ibaraki 300-0393, Japan
}

AdDitional INDEX words. cultivar identification, fruit tree, microsatellite, parentage analysis, simple sequence repeat

\begin{abstract}
To develop and characterize valid simple sequence repeat (SSR) markers in chestnut (Castanea spp.), we used a selective hybridization method to perform SSR sequence enrichment in the genomic library of chinese chestnut (Castanea mollissima). From 47 sequences of the enriched library, we designed 24 primer pairs for SSR polymerase chain reaction (PCR). SSR PCR was performed for 23 chinese chestnut cultivars. Among the 24 primers, 22 primers amplified their respective SSR loci; 21 primer pairs yielded polymorphic fragments across the cultivars. Among these 21 primer pairs, 17 primer pairs amplified single SSR loci with polymorphisms. Multilocus amplification patterns were observed in the other four primers. For the corresponding 17 SSR loci, the number of alleles per locus was two to 13, and the average number of alleles was 7.19. The observed heterozygosity (number of genotypes of the heterozygote/total number of genotypes scored at that locus) ranged from 0.00 to 0.87 (average $=0.68$ ), and the expected heterozygosity ranged from 0.00 to 0.87 (average $=0.68$ ). One of the SSR loci-ICMA014-was a unique locus, because the primer pair for this locus did not amplify any fragment in any of the cultivars, except in Qianchi, which was used to construct the SSR-enriched genomic library. Cross-species amplifications using the 17 primer pairs were also successful in the other species of genus Castanea. A dendrogram depicting the relationships among the genotypes on the basis of genetic distances was generated using the unweighted pair group method with arithmetic mean cluster analysis. The genotypes were clearly separated into three large groups, which reflected the three cultivated species, namely, $C$. mollissima, $C$. crenata, and $C$. sativa.
\end{abstract}

Chestnut is one of the most important nut trees in the world and has been cultivated for more than 2700 years in Asia (Adua, 1999). Castanea species have a diploid genome $(\mathrm{n}=12)$ and are distributed in the temperate regions. The genus Castanea is a member of the Fagaceae family and comprises 10 species, including three cultivated species, namely, chinese chestnut (C. mollissima), japanese chestnut (C. crenata), and european chestnut (C. sativa) (U.S. Department of Agriculture, 2007). Among these, chinese chestnut and japanese chestnut are the two most economically important Castanea species (Tanaka and Kotobuki, 1992). Chinese chestnut originated and is mainly cultivated in China.

Several molecular markers, including isozyme markers (Casasoli et al., 2001; Dane et al., 2003; Sawano et al., 1984), chloroplast sequences (Fineschi et al., 2000), random amplified polymorphic DNA markers (Casasoli et al., 2001; Galderisi et al., 1998; Inoue et al., 2003; Morimoto et al., 1997), inter-simple sequence repeat markers (Casasoli et al., 2001), restriction fragment length polymorphism markers (Morimoto et al., 1997), and amplified fragment length polymorphism markers (Yamamoto et al., 1998), have been used to study the genetic

Received for publication 7 Nov. 2007. Accepted for publication 23 Oct. 2009. We thank Mr. Kazuo Kotobuki, National Institute of Fruit Tree Science, Japan, for help in collecting leaf samples used in this work.

${ }^{1}$ Corresponding author. E-mail: einoue@mx.ibaraki.ac.jp. diversity and relationships in chestnut cultivars. Simple sequence repeat (SSR), which is one such marker, is a short tandem repeat sequence that is distributed throughout the genome. They are used to develop molecular markers for several organisms such as horticultural crops (Clarke and Tobutt, 2003, 2006; Inoue et al., 2006; James et al., 2003; Yamamoto et al., 2002; Zane et al., 2002). Recently, SSR markers have been developed (Buck et al., 2003; Yamamoto et al., 2003) and used not only for genetic differentiation, which is used in phylogenetic research and cultivar identification, but also for advanced genetic mapping, including comparative mapping (Barreneche et al., 2003) and quantitative trait locus (QTL) analysis (Casasoli et al., 2004, 2006), in chestnut plants of different origins. SSRs afford a high level of polymorphism and codominance. Although the development of SSR markers is difficult, they can be easily detected using polymerase chain reaction (PCR) and electrophoresis. Microsatellite markers for japanese chestnuts and european chestnuts have been developed and used (Buck et al., 2003; Yamamoto et al., 2003); however, there have been no studies on the development of microsatellite markers for chinese chestnuts. Furthermore, the number of available markers is insufficient for breeding selection or advanced QTL analysis in chestnut, and the available types of repeat motifs are limited.

Therefore, in this study, to develop an analysis tool and obtain information regarding the chestnut genome, we attempted 
to develop useful microsatellite markers from an enriched genomic library of chinese chestnut that was constructed by performing selective hybridization with eight types of microsatellite repeat sequences (Zane et al., 2002).

We also investigated the use of these markers in cultivar identification and parentage analysis. Furthermore, we characterized the microsatellite markers in Castanea and investigated their cross-species transferability to determine whether the markers were effective in characterizing other members of the Fagaceae family.

\section{Materials and Methods}

Plant materials. A microsatellite-enriched genomic library was constructed from the genomic DNA of the chinese chestnut 'Qianchi'. 'Qianchi' is an old cultivar grown in Hubei Province, China. To survey the SSR loci in Castanea, 23 chinese chestnut accessions, 42 japanese chestnut accessions, one european chestnut accession, and five interspecific hybrids were used as the plant materials (Table 1). To investigate the cross-species transferability of the SSR primers developed in this experiment, we used related woody plants of the Fagaceae family, including 17 species belonging to four different genera.

DNA Extraction. Mature leaves of each genotype were frozen in liquid nitrogen and ground with tungsten beads by using a tissue grinder (SH-48; Kurabo, Osaka, Japan). Genomic DNA was isolated from the ground tissue by performing a modified protocol of the cetyltrimethylammonium bromide method (Bousquet et al., 1990). The extracted DNA was quantified by ultraviolet spectrophotometry with the Molecular Probes $^{\mathrm{TM}}$ PicoGreen dsDNA Quantitation Reagent (Invitrogen, Carlsbad, CA).

DEVElopment OF SIMPLE SEQUENCE REPEAT MARKERS. SSR sequences were obtained from the 'Qianchi' genomic library

Table 1. Chestnut accessions used for characterizing the newly developed simple sequence repeat (SSR) loci in Castanea. ${ }^{\mathrm{z}}$

\begin{tabular}{|c|c|c|c|c|c|c|c|}
\hline No. & Accessions & Castanea species & Origin & No. & Accessions & Castanea species & Origin \\
\hline 1 & Chushuhong & C. mollissima & China & 36 & Shougatu & C. crenata & Japan \\
\hline 3 & Ershuizhao & C. mollissima & China & 38 & Arima & C. crenata & Japan \\
\hline 5 & Hongguang & C. mollissima & China & 40 & Ginrei & C. crenata & Japan \\
\hline 6 & Hongli & C. mollissima & China & 41 & Kanotsume & C. crenata & Japan \\
\hline 7 & Honglilhao & C. mollissima & China & 42 & Hashikami & C. crenata & Japan \\
\hline 10 & Linfen & C. mollissima & China & 45 & Wasechoubei & C. crenata & Japan \\
\hline 11 & Niandiban & C. mollissima & China & 46 & Ishiduka & C. crenata & Japan \\
\hline 12 & Qianchi & C. mollissima & China & 47 & Kasaharawase & C. crenata & Japan \\
\hline 13 & Shandongzicui & C. mollissima & China & 48 & Choukouji & C. crenata & Japan \\
\hline 14 & Shandongzicui3 & C. mollissima & China & 49 & Obuse3gou & C. crenata & Japan \\
\hline 19 & Xinlong9hao & C. mollissima & China & 54 & Osaya & C. crenata & Japan \\
\hline 20 & Yanchen207 & C. mollissima & China & 55 & Imakita & C. crenata & Japan \\
\hline 21 & Yebanli & C. mollissima & China & 56 & Ibuki & C. crenata & Japan \\
\hline 22 & Yebianzha & C. mollissima & China & 57 & Autumn koron & C. crenata & Japan \\
\hline 23 & Yelichang & C. mollissima & China & 58 & Autumn poron & C. crenata & Japan \\
\hline 24 & Idumo & C. crenata & Japan & 59 & Togenasiguri & C. crenata & Japan \\
\hline 25 & Isiduti & C. crenata & Japan & 60 & Shibaguri1 & C. crenata & Japan \\
\hline 26 & Kinka & C. crenata & Japan & 61 & Shibaguri2 & C. crenata & Japan \\
\hline 27 & Moriwase & C. crenata & Japan & 62 & Shibaguri3 & C. crenata & Japan \\
\hline 28 & Kotai & C. crenata & Japan & 63 & Kunimi & C. crenata & Japan \\
\hline 29 & Banseki & C. crenata & Japan & 64 & Tanzawa & C. crenata & Japan \\
\hline 35 & & & & 71 & AW-7 & C. crenata $\times$ C. sativa & Japan \\
\hline
\end{tabular}

zTwenty-three chinese chestnut accessions, 42 japanese chestnut accessions, one european chestnut accession, and five interspecific hybrids were used. The genomic DNA for SSR identification was extracted from the mature leaves of the accessions. 
that was enriched for eight different types of microsatellites(GA)n, (GT)n, (GC)n, (AC)n, (CAA)n, (GCC)n, (CTG)n, and (CAG)n-by selective hybridization (Zane et al., 2002). The genomic DNA of 'Qianchi' was digested with RsaI (New England Biolabs, Ipswich, MA) at $37{ }^{\circ} \mathrm{C}$ for $\approx 12 \mathrm{~h}$. The digested DNA fragments were ligated to the adapter DNA that was constructed using ad1-5'-CTCTTGCTTACGCGTG GACTA-3' and ad2-5'-TAGTCCACGCGTAAGCAAGAG CACA-3' . The ligation reaction was performed using T4 DNA ligase (Promega, Madison, WI) at room temperature for $2 \mathrm{~h}$. Then, the fragments were incubated at $4{ }^{\circ} \mathrm{C}$ overnight. The DNA fragments and the adapters were amplified by PCR with ad1 as the primer. PCR was performed using a $50-\mu \mathrm{L}$ sample mixture containing $5 \mu \mathrm{L}$ of the ligated products, $1 \times$ PCR buffer (without magnesium), $1.5 \mathrm{~mm} \mathrm{MgCl}_{2}, 1 \mu \mathrm{M}$ ad1 primer, $0.2 \mathrm{~mm}$ of each dNTP, and 2U Taq DNA polymerase (Invitrogen) in a thermocycler (model 9700; Applied Biosystems, Foster City, CA). The following protocol was used for PCR: 35 cycles of $40 \mathrm{~s}$ at $95^{\circ} \mathrm{C}, 1 \mathrm{~min}$ of annealing at $60^{\circ} \mathrm{C}$, and $3 \mathrm{~min}$ of extension at $72{ }^{\circ} \mathrm{C}$. Subsequently, the amplified fragments were hybridized with eight types of SSR oligonucleotides: (GA)17, (GT)17, (GC)17, (AC)17, (CAA)10, (GCC)10, (CTG)10, and (CAG)10. The oligonucleotides were bound to a positively charged nylon membrane (Hybond-N+; GE Healthcare, Amersham, UK), and the amplified fragments were enriched for genomic DNA containing the previously mentioned SSR sequences.

The enriched DNA was amplified by PCR and desalted using a Suprec TM-02 centrifuge device (Takara Bio, Madison, WI). Then, the products were ligated with the pGEM-T Easy Vector (Promega) and used to transform Escherichia coli DH5 $\alpha$ cells. The positive clones, which contained the plasmid DNA with an $\approx 450$-bp-long DNA insert, were selected by direct colony PCR. The plasmids were extracted, and their cloning sites were sequenced using a Big Dye version 3.1 cycle sequencing kit and an ABI3100 genetic analyzer (Applied Biosystems). On the basis of the SSR-containing sequences, the primer pairs for sequences on both sides of the SSR region were constructed using PRIMER3 (Rozen and Skaletsky, 2000). One primer of each pair was labeled using the fluorescent dye 6-FAM or VIC.

Simple SEQUENCE REPEAT POLYMERASE CHAIN REACTION AND ELECTROPHORESIS USING A CAPILLARY SEQUENCER. SSR PCR WAS performed using $12.5-\mu \mathrm{L}$ reaction mixtures containing $0.2 \mathrm{mM}$ of each dNTP, $1 \times$ PCR buffer, $1.5 \mathrm{mM} \mathrm{MgCl}_{2}, 0.25 \mathrm{U}$ Taq DNA polymerase (Invitrogen), $0.2 \mu \mathrm{M}$ of each primer, and $6.25 \mathrm{ng}$ of genomic DNA. The amplification reactions were performed using the following protocol: denaturation at $94{ }^{\circ} \mathrm{C}$ for $3 \mathrm{~min}$; 35 cycles of amplification at $94{ }^{\circ} \mathrm{C}$ for $30 \mathrm{~s}, 45 \mathrm{~s}$ of annealing at $55^{\circ} \mathrm{C}$ or $60{ }^{\circ} \mathrm{C}, 1 \mathrm{~min}$ of extension at $72{ }^{\circ} \mathrm{C}$, and a final extension step at $72{ }^{\circ} \mathrm{C}$ for $10 \mathrm{~min}$. The amplification products were separated using an ABI3100 sequencer with the GENESCAN and GENOTYPER (Applied Biosystems) software packages and a GS500LIZ ladder (Applied Biosystems).

ANAlysis of SIMPle SEQUENCE REPEAT LOCI. The SSR alleles were named according to their length at each locus. The expected heterozygosity (Nei, 1973), observed heterozygosity, and the probability of identity (Paetkau et al., 1995) of the 17 microsatellites that were associated with single loci were calculated using IDENTITY1.0 (Wagner and Sefc, 1999). GENEPOP (Raymond and Rousset, 1995) was used to identify deviations from the Hardy-Weinberg equilibrium (HWE), and the linkage disequilibrium (LD) within populations was analyzed using the Markov chain parameters. Pairwise genetic distances, which were determined on the basis of the proportion of shared alleles, were calculated for all the genotypes. A dendrogram was generated using the unweighted pair group method with arithmetic mean (UPGMA) cluster analysis method, which uses the PHYLIP3.6 program (Felsenstein, 2005) and the TreeView software (Page, 1996).

\section{Results}

DEVELOPMENT OF THE SIMPLE SEQUENCE REPEAT-ENRICHED LIBRARY AND THE GENOMIC SEQUENCES CONTAINING SIMPLE SEQUENCE REPEAT REGIONS. A total of 141 SSR-enriched clones were constructed from the nuclear genome of the 'Qianchi' by performing the selective hybridization method, and 105 positive clones were selected from the library by colony direct PCR. Among these, 47 positive clones that contained plasmids with $\approx 450$-bp-long DNA inserts were sequenced. Among the selected clones, 41 clones, including four clones with overlapping sequences, were found to contain SSR sequences.

In the 37 unique sequences, 57 SSR regions were identified: $39 \mathrm{GA} / \mathrm{CT}$ regions $(68.4 \%), 14 \mathrm{GT} / \mathrm{CA}$ regions $(24.6 \%)$, two $\mathrm{CAG} / \mathrm{GTC}$ regions $(3.5 \%)$, one $\mathrm{GCC} / \mathrm{CGG}$ region $(1.8 \%)$, and one AT/TA region (1.8\%). To obtain the primers, we designed 24 primer pairs for SSR PCR using the 37 unique clones, excluding the clones that had very short flanking sequences.

EVALUATION OF THE SIMPLE SEQUENCE REPEAT PRIMERS IN THE CHINESE CHESTNUT CULTIVARS. The 24 newly developed microsatellite primer pairs were evaluated using 23 chinese chestnut cultivars (Table 1). The results of this evaluation are shown in Table 2 .

Among these pairs, 22 primer pairs amplified their respective SSR loci in the chinese chestnut cultivars. We assessed the occurrence of polymorphic fragments from these 22 primers. Among these primer pairs, 17 amplified single polymorphic SSR loci (Table 2). One primer pair (ICMA001) amplified only a nonpolymorphic fragment in 23 accessions. Four primers-ICMA006, ICMA012, ICMA017, and ICMA024-amplified more than three alleles per locus, thereby generating multiple loci patterns. The analyses with the 17 SSR primers that amplified single loci yielded 129 putative alleles, including one null allele. The number of alleles per locus varied from two to 13 , and the average number of alleles was 7.19. The observed heterozygosity, which is the ratio of the number of heterozygotic genotypes to the total number of genotypes scored at the locus, ranged from 0.00 to 0.87 (average, 0.68). The expected heterozygosity ranged from 0.00 to 0.87 (average, 0.68 ). Although there were no significant deviations from $\mathrm{HWE}$ at $P<0.01$, at $P<0.05$, there were significant deviations in three loci, namely, ICMA004, ICMA019, and ICMA022 (Table 2). The LD between ICMA008 and ICMA019 was evaluated $(P>0.001)$.

Cross-species transferability. Cross-species amplifications using the 17 primer pairs were successful in other species of genus Castanea. Among the 17 SSR loci in the 42 japanese chestnut accessions, the number of alleles per locus was two to 21 (average, 6.91) (Table 2). The observed heterozygosity ranged from 0.17 to 0.93 (average, 0.59), and the expected heterozygosity ranged from 0.18 to 0.93 (average, 0.58 ). The SSR markers developed from chinese chestnut exhibited high polymorphism. Therefore, they can be used as a reliable tool for differentiating among the japanese chestnut accessions. Among the 42 japanese chestnut accessions, significant deviations from 
Table 2. Sequences of the 22 simple sequence repeat (SSR) primers developed from chinese chestnut and their characterizations in 23 chinese chestnut cultivars and 42 japanese chestnut cultivars, including information on allele number, expected heterozygosity $\left(\mathrm{H}_{\mathrm{E}}\right)$, observed heterozygosity $\left(\mathrm{H}_{\mathrm{O}}\right)$, and probability of identity $(\mathrm{PI}){ }^{\mathrm{z}}$

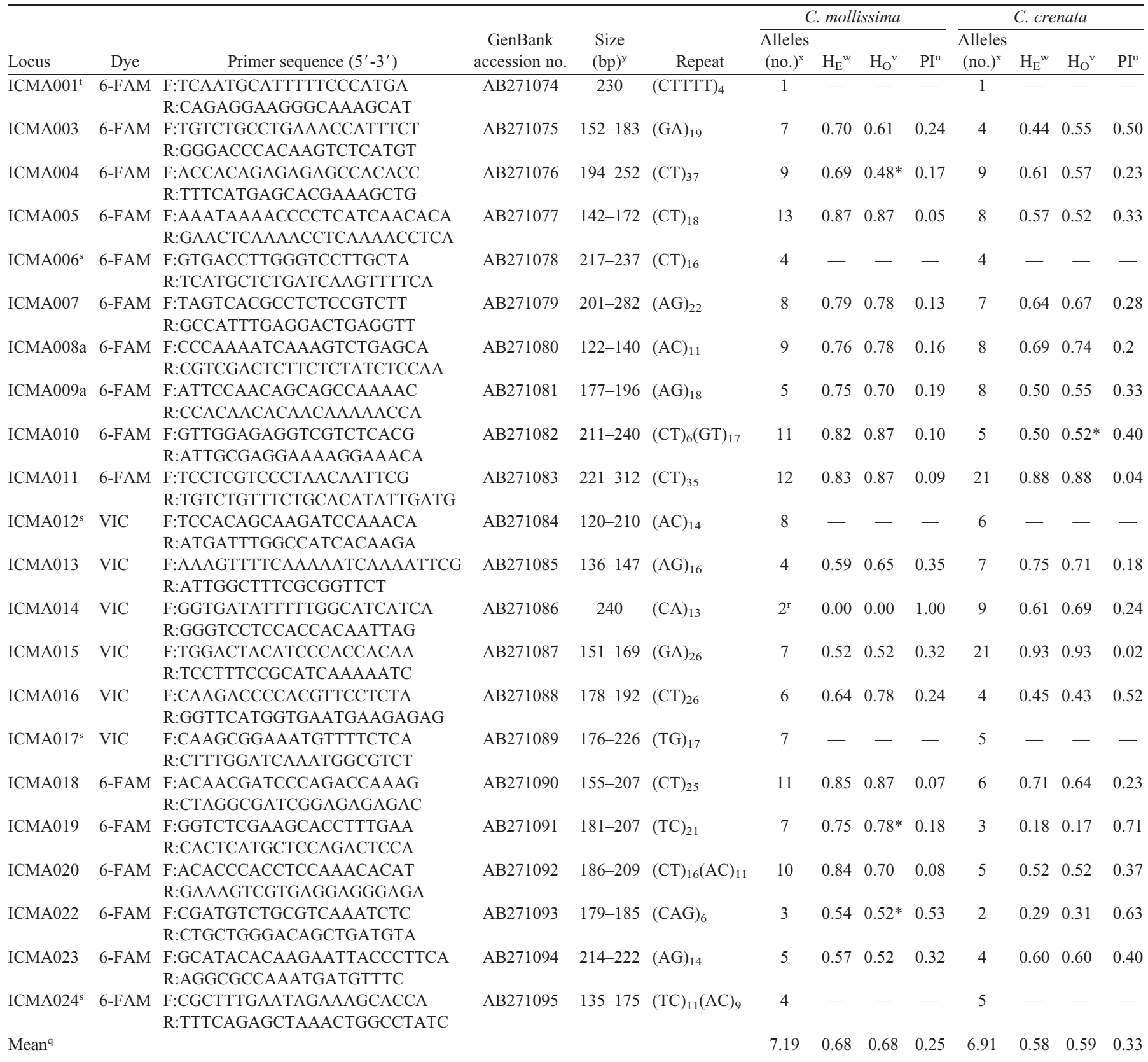

${ }^{\mathrm{z} S S R}$ polymerase chain reaction was conducted using a fluorescent-labeled forward primer, and the amplification products were subsequently separated using an ABI3100 genetic analyzer with the GENESCAN and GENOTYPER software and the GS500LIZ ladder (Applied Biosystems, Foster City, CA).

${ }^{\mathrm{y}}$ Size range of single SSR locus with polymorphisms observed in 23 cultivars of chinese chestnuts.

${ }^{x}$ Allele numbers detected in the species.

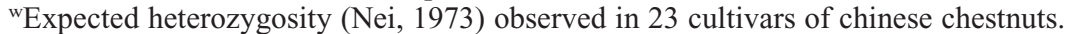

'Observed heterozygosity (direct count heterozygosity) observed in 23 cultivars of chinese chestnuts. Significant departures from the HardyWeinberg equilibrium, $* P<0.05$.

uProbability of identity (Paetkau et al., 1995) observed in 23 cultivars of chinese chestnuts.

${ }^{t} \mathrm{H}_{\mathrm{E}}, \mathrm{H}_{\mathrm{O}}$, and PI were not calculated because the locus did not segregate.

${ }^{\mathrm{s}} \mathrm{H}_{\mathrm{E}}, \mathrm{H}_{\mathrm{O}}$, and PI were not calculated because more than three alleles were observed per locus.

${ }^{\mathrm{r}}$ Including a null allele.

${ }^{\mathrm{q}}$ Calculated by 17 single SSR locus with polymorphisms. 
the HWE were detected only in ICMA010 at $P<0.05$ (Table 2). Linkage disequilibria were detected between ICMA004 and ICMA011, ICMA007 and ICMA015, ICMA009 and ICMA015, and ICMA008 and ICMA018 $(P>0.001)$.

In the european chestnut accessions, 15 primers, except ICMA005 and ICMA014, amplified one or two visible fragments. At seven loci in the european chestnut accessions, one or two alleles were species-specific (Table 3). The 17 primers were able to amplify polymorphic fragments in 17 species belonging to the Fagaceae family (Table 4). ICMA003 and ICMA022 amplified one or two clear bands in all the tested genotypes in the Fagaceae family.

Characterization OF THE ICMA014 locus With A NUlL ALlELE IN CHINESE Chestnut. The SSR locus ICMA014 is considered a unique locus, because it did not amplify any fragment in any of the chinese chestnut cultivars, except in Qianchi, which was used to develop the genomic library. Interestingly, the ICMA014 primer could produce one or two amplified fragments in all the japanese chestnut accessions, and nine putative alleles for this locus were confirmed in the 42 japanese chestnut accessions (Table 3). Furthermore, we used $\approx 100$ chinese chestnut accessions to investigate this locus; however, the amplification of the locus was confirmed in only in a few cultivars, which were closely related to 'Qianchi' (data not shown). These results indicated the existence of a null allele in the ICMA014 locus in chinese chestnut. To confirm this hypothesis, we examined the interspecific cultivar Riheiguri and its offspring. Because 'Riheiguri' is an interspecific hybrid of japanese chestnut and chinese chestnut, its genotype on the ICMA014 locus is expected to be $244 / 244$ or $244 / n$. Furthermore, a cross between the japanese chestnut cultivar Toyotamawase (genotype, 242/242) and 'Riheiguri' yielded an offspring cultivar known as Gakuou. If 'Riheiguri' contains a null allele at the ICMA014 locus inherited from the chinese parent, its genotype will be $244 / \mathrm{n}$. Consequently, the expected genotype of 'Gakuou' will be $242 / 244$ or $242 / \mathrm{n}$. Because 'Gakuou' exhibits only a single 242 allele, we concluded that its genotype is $242 / \mathrm{n}$, and it possesses a null allele (Fig. 1).

Genetic ChaRACTERIZATION OF THE GenOtyPes in CASTANEA. To depict the relationships among the genotypes, we generated a dendrogram using the genetic distances (Fig. 2). The genotypes were clearly divided into three large groups, which reflected the three species: $C$. mollissima, $C$. crenata, and $C$. sativa (Fig. 2). In the japanese chestnut accessions, the cultivar pairs Nakatetanba and Wasechoubei, Chuutan A and Ginrei, and Ishiduka and Tamanishiki had identical genotypes at all 17 loci.

\section{Discussion}

In the present study, we used the selective hybridization method to develop 17 polymorphic microsatellite primers from an enriched genomic library of chinese chestnut. The average observed and expected heterozygosities were higher than 0.6 , thereby indicating a high degree of variation in the isolated loci and the absence of null alleles. This is the first report on the development of microsatellite markers for chinese chestnut.

In this study, more than $87 \%$ of the clones from the enriched genomic library were found to contain microsatellite sequences. This value for the occurrence of microsatellites in the positive sequences is higher than that reported in previous studies (Buck et al., 2003; Yamamoto et al., 2003). Furthermore, we obtained similar results in our previous study on the development of microsatellite markers in japanese pear (Pyrus pyrifolia) (Inoue et al., 2006). Because the selective hybridization method has high concentration efficiency and is

Table 3. Allelic distribution of the 17 simple sequence repeat loci in 23 chinese chestnut accessions, 42 japanese chestnut accessions, and one european chestnut accession. ${ }^{\mathrm{z}}$

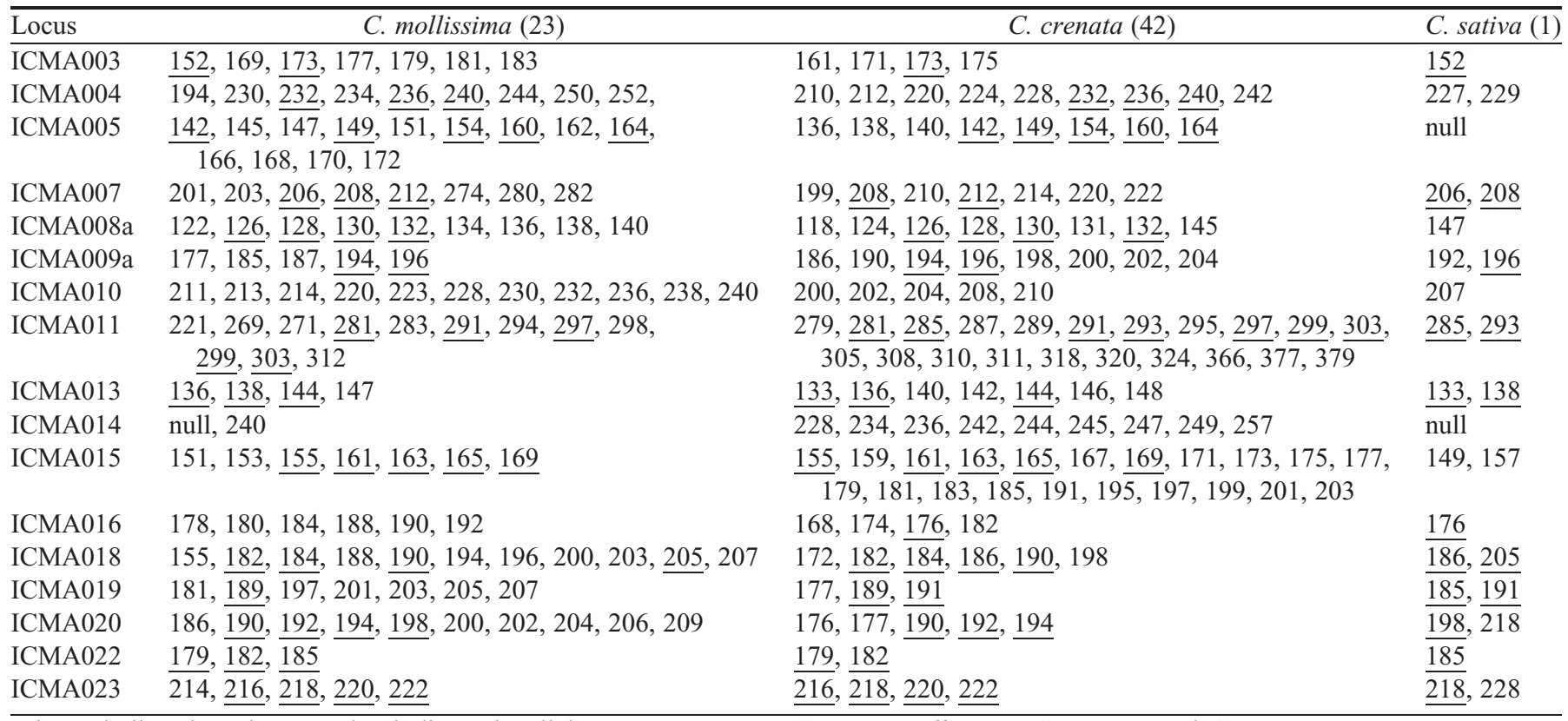

${ }^{\mathrm{z}}$ The underlines have been used to indicate the alleles common among Castanea mollissima, C. crenata, and C. sativa. 

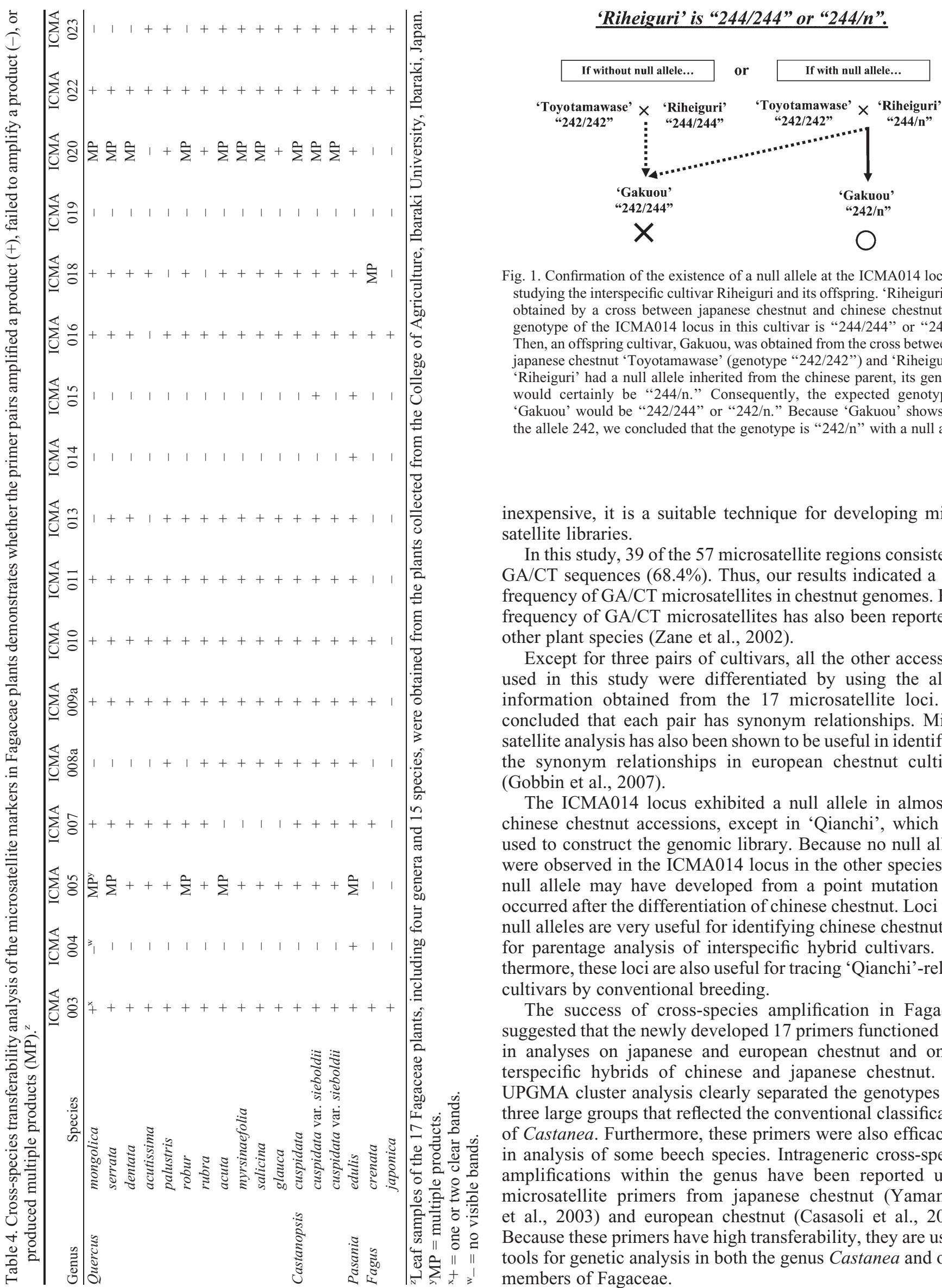

Fig. 1. Confirmation of the existence of a null allele at the ICMA014 locus by studying the interspecific cultivar Riheiguri and its offspring. 'Riheiguri' was obtained by a cross between japanese chestnut and chinese chestnut. The genotype of the ICMA014 locus in this cultivar is " $244 / 244$ " or " $244 / n$." Then, an offspring cultivar, Gakuou, was obtained from the cross between the japanese chestnut 'Toyotamawase' (genotype "242/242") and 'Riheiguri'. If 'Riheiguri' had a null allele inherited from the chinese parent, its genotype would certainly be " $244 / \mathrm{n}$." Consequently, the expected genotype of "Gakuou' would be " $242 / 244$ " or " $242 / n$." Because 'Gakuou' shows only the allele 242 , we concluded that the genotype is " $242 / n$ " with a null allele.

inexpensive, it is a suitable technique for developing microsatellite libraries.

In this study, 39 of the 57 microsatellite regions consisted of GA/CT sequences (68.4\%). Thus, our results indicated a high frequency of $\mathrm{GA} / \mathrm{CT}$ microsatellites in chestnut genomes. High frequency of GA/CT microsatellites has also been reported in other plant species (Zane et al., 2002).

Except for three pairs of cultivars, all the other accessions used in this study were differentiated by using the allelic information obtained from the 17 microsatellite loci. We concluded that each pair has synonym relationships. Microsatellite analysis has also been shown to be useful in identifying the synonym relationships in european chestnut cultivars (Gobbin et al., 2007).

The ICMA014 locus exhibited a null allele in almost all chinese chestnut accessions, except in 'Qianchi', which was used to construct the genomic library. Because no null alleles were observed in the ICMA014 locus in the other species, the null allele may have developed from a point mutation that occurred after the differentiation of chinese chestnut. Loci with null alleles are very useful for identifying chinese chestnut and for parentage analysis of interspecific hybrid cultivars. Furthermore, these loci are also useful for tracing 'Qianchi'-related cultivars by conventional breeding.

The success of cross-species amplification in Fagaceae suggested that the newly developed 17 primers functioned well in analyses on japanese and european chestnut and on interspecific hybrids of chinese and japanese chestnut. The UPGMA cluster analysis clearly separated the genotypes into three large groups that reflected the conventional classification of Castanea. Furthermore, these primers were also efficacious in analysis of some beech species. Intrageneric cross-species amplifications within the genus have been reported using microsatellite primers from japanese chestnut (Yamamoto et al., 2003) and european chestnut (Casasoli et al., 2006). Because these primers have high transferability, they are useful tools for genetic analysis in both the genus Castanea and other members of Fagaceae. 


\section{Literature Cited}

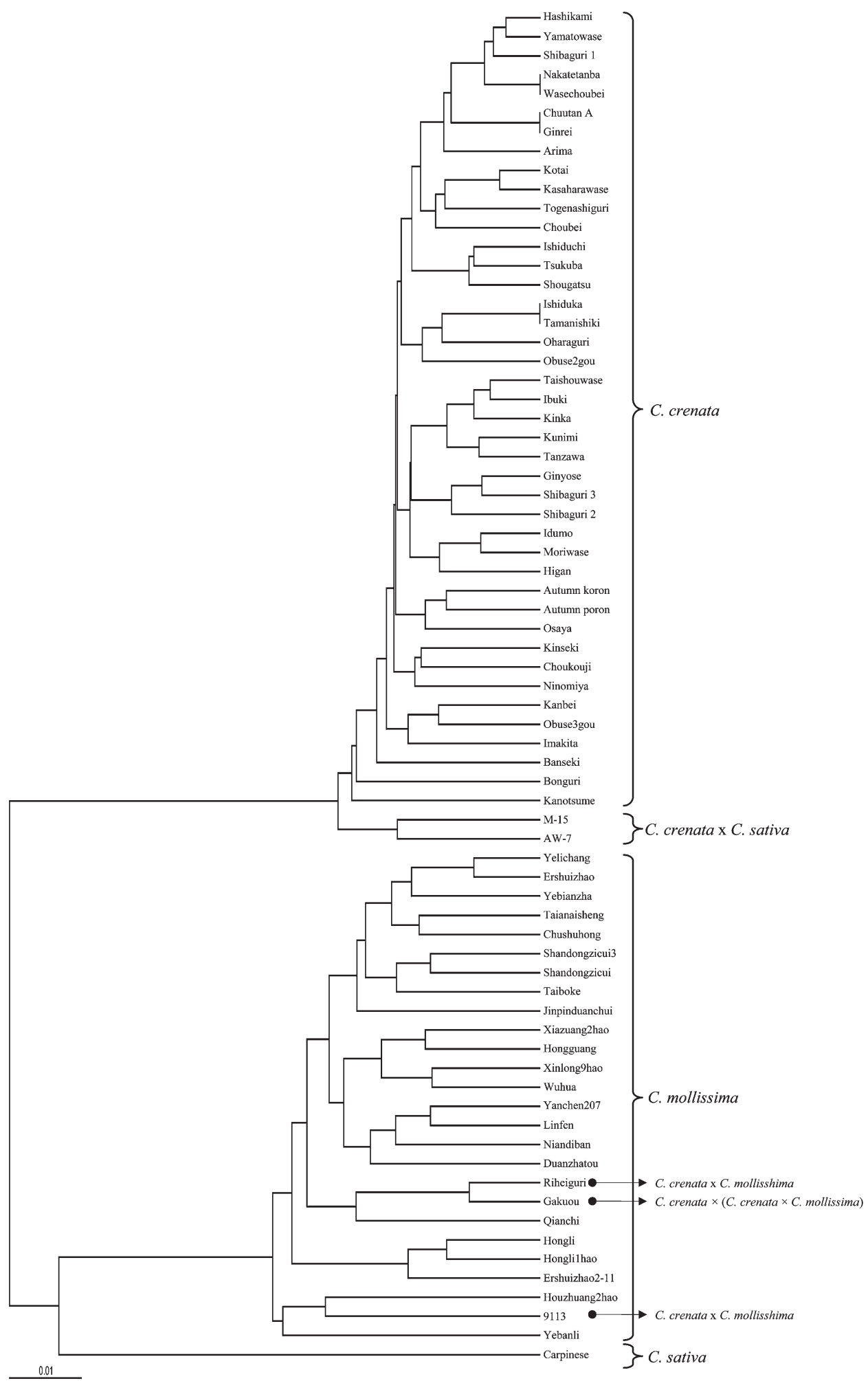

Fig. 2. Dendrogram generated from the unweighted pair group method with arithmetic mean cluster analysis using PHYLIP3.6 programs (Felsenstein, 2005); the dendrogram is based on the genetic distances of the 17 microsatellite loci in the chestnut genome, including those in 23 chinese chestnut accessions, 42 japanese chestnut accessions, one european chestnut accession, and five interspecific hybrids. The tree was developed using the TreeView software (Page, 1996). Almost all the accessions used in this study were separated in the dendrogram, except for three pairs of cultivars of japanese chestnut. A total of 66 genotypes without five interspecific hybrids were clearly separated into three large groups that reflected the three distinct species: Castanea mollissima, C. crenata, and C. sativa.
Adua, M. 1999. The sweet chestnut throughout history from the Miocene to the third millennium. Acta Hort. 494:29-36.

Barreneche, T., M. Casasoli, K. Russell, A. Akkak, H. Meddour, C. Plomion, F. Villani, and A. Kremer. 2003. Comparative mapping between Quercus and Castanea using simple-sequence repeats (SSRs). Theor. Appl. Genet. 108:558-566.

Bousquet, J., L. Simon, and M. Lalonde. 1990. DNA amplification from vegetative and sexual tissues of tree using polymerase chain reaction. Can. J. For. Res. 20:254-257.

Buck, E.J., M. Hadonou, C.J. James, D. Blakesley, and K. Russell. 2003. Isolation and characterization of polymorphic microsatellites in european chestnut (Castanea sativa Mill.). Mol. Ecol. Notes 3:239-241.

Casasoli, M., J. Derory, C. MoreraDutrey, O. Brendel, I. Porth, J.M. Guehl, F. Villani, and A. Kremer. 2006. Comparison of quantitative trait loci for adaptive traits between oak and chestnut based on an expressed sequence tag consensus map. Genetics 172:533-546.

Casasoli, M., C. Mattioni, M. Cherubini, and F. Villani. 2001. A genetic linkage map of european chestnut (Castanea sativa Mill.) based on RAPD, ISSR and isozyme markers. Theor. Appl. Genet. 102:1190-1199.

Casasoli, M., D. Pot, C. Plomion, M.C. Monteverdi, T. Barreneche, M. Lauteri, and F. Villani. 2004. Identification of QTLs affecting adaptive traits in Castanea sativa Mill. Plant Cell Environ. 27:1088-1101.

Clarke, J.B. and K.R. Tobutt. 2003. Development and characterization of polymorphic microsatellites from Prunus avium 'Napoleon'. Mol. Ecol. Notes 3:578-580.

Clarke, J.B. and K.R. Tobutt. 2006. Development of microsatellite primers and two multiplex polymerase chain reactions for the common elder (Sambucus nigra). Mol. Ecol. Notes 6:453455.

Dane, F., P. Lang, H. Huang, and Y. Fu. 2003. Intercontinental genetic divergence of Castanea species in eastern Asia and eastern North America. Heredity 91:314-321.

Felsenstein, J. 2005. PHYLIP (Phylogeny Inference Package). Version 3.6. Dept. Genome Sciences, University of Washington, Seattle, WA.

Fineschi, S., D. Taurchini, F. Villani, and G.G. Vendramin. 2000. Chloroplast DNA polymorphism reveals little geographical structure in Castanea 
sativa Mill. (Fagaceae) throughout southern european countries. Mol. Ecol. 9:1495-1503.

Galderisi, U., M. Cipollaro, G.D. Bernardo, L.D. Masi, G. Galano, and A. Cascino. 1998. Molecular typing of Italian sweet chestnuts cultivars by random amplified polymorphic DNA analysis. J. Hort. Sci. Biotechnol. 73:259-263.

Gobbin, D., L. Hohl, L. Conza, M. Jermini, C. Gessler, and M. Conedera. 2007. Microsatellite-based characterization of the Castanea sativa cultivar heritage of southern Switzerland. Genome 50:1089-1103.

Inoue, E., H. Hara, S. Ruan, M. Sano, and T. Tsukihashi. 2003. Origin and RAPD analysis of japanese chestnut (Castanea crenata Sieb. et Zucc.) cultivated in china. Hort. Res. 2:253-257 [in Japanese].

Inoue, E., Y. Matsuki, H. Anzai, and K. Evans. 2006. Isolation and characterization of microsatellite markers in japanese pear (Pyrus pyrifolia Nakai). Mol. Ecol. Notes 7:445-447.

James, C.M., F. Wilson, A.M. Hadonou, and K.R. Tobutt. 2003. Isolation and characterization of polymorphic microsatellites in diploid strawberry (Fragaria vesca L.) for mapping, diversity studies and clone identification. Mol. Ecol. Notes 3:171-173.

Morimoto, Y., T. Shimada, T. Ozaki, K. Nomura, H. Araki, and M. Yoshida. 1997. Identification and relationship of japanese chestnut varieties by RAPD and southern blot analysis. Sci. Rpt. Faculty Agr. Kobe Univ. 22:63-69.

Nei, M. 1973. Analysis of gene diversity in subdivided populations. Proc. Natl. Acad. Sci. USA 70:3321-3323.

Paetkau, D., W. Calvert, I. Stirling, and C. Strobeck. 1995. Microsatellite analysis of population structure in Canadian polar bears. Mol. Ecol. 4:347-354.

Page, R.D.M. 1996. TREEVIEW: An application to display phylogenetic trees on personal computers. Comput. Appl. Biosci. 12:357-358.
Raymond, M. and F. Rousset. 1995. GENEPOP (version 1.2): Population genetics software for exact tests and ecumenicism. J. Hered. 86:248-249.

Rozen, S. and H.J. Skaletsky. 2000. PRIMER3 on the WWW for general users and for biologist programmers, p. 365-386. In: S. Krawetz and S. Misener (eds.). Bioinformatics methods and protocols: Methods in molecular biology. Humana Press, Totowa, NJ.

Sawano, M., T. Ichii, T. Nakanishi, and Z. Kotera. 1984. Studies on identification of chestnut species and varieties by isozyme analysis. Sci. Rpt. Faculty Agr. Kobe Univ. 16:67-71.

Tanaka, K. and K. Kotobuki. 1992. Studies on peeling characteristics of japanese chestnut (Castanea crenata Sieb. et Zucc.), chinese chestnut (C. mollissima BL.) and their hybrids. Acta Hort. 317:175180.

U.S. Department of Agriculture. 2007. Germplasm Resources Information Network (GRIN). 11 Nov. 2007. <http://www.ars-grin.gov/ cgi-bin/npgs/html/taxgenform.pl>.

Wagner, H.W. and K.M. Sefc. 1999. IDENTITY 1.0. Centre for Applied Genetics, University of Agricultural Sciences, Vienna, Austria.

Yamamoto, T., T. Kimura, M. Shoda, Y. Ban, T. Hayashi, and N. Matsuta. 2002. Development of microsatellite markers in the japanese pear (Pyrus pyrifolia Nakai). Mol. Ecol. Notes 2:14-16.

Yamamoto, T., T. Shimada, K. Kotobuki, Y. Morimoto, and M. Yoshida. 1998. Genetic characterization of Asian chestnut varieties assessed by AFLP. Breed. Sci. 48:359-363.

Yamamoto, T., T. Tanaka, K. Kotobuki, N. Matsuta, M. Suzuki, and Y. Hayashi. 2003. Characterization of simple sequence repeats in japanese chestnut. J. Hort. Sci. Biotechnol. 78:197-203.

Zane, L., L. Bargelloni, and T. Patarnello. 2002. Strategies for microsatellite isolation: A review. Mol. Ecol. 11:1-16. 